\title{
A mysterious giant ichthyosaur from the lowermost Jurassic of Wales
}

Jeremy E. Martin, Peggy Vincent, Guillaume Suan, Tom Sharpe, Peter Hodges, Matt Williams, Cindy Howells, and Valentin Fischer

Acta Palaeontologica Polonica 60 (4), 2015: 837-842 doi:http://dx.doi.org/10.4202/app.00062.2014

Ichthyosaurs rapidly diversified and colonised a wide range of ecological niches during the Early and Middle Triassic

period, but experienced a major decline in diversity near the end of the Triassic. Timing and causes of this demise and the subsequent rapid radiation of the diverse, but less disparate, parvipelvian ichthyosaurs are still unknown, notably because of inadequate sampling in strata of latest Triassic age. Here, we describe an exceptionally large radius from Lower Jurassic deposits at Penarth near Cardiff, south Wales (UK) the morphology of which places it within the giant Triassic shastasaurids. A tentative total body size estimate, based on a regression analysis of various complete ichthyosaur skeletons, yields a value of 12-15 $\mathrm{m}$. The specimen is substantially younger than any previously reported last known occurrences of shastasaurids and implies a Lazarus range in the lowermost Jurassic for this ichthyosaur morphotype.

Jeremy E. Martin [jeremy.martin@ens-lyon.fr], School of Earth Sciences, University of Bristol, Wills Memorial Building, Queen's Road, Bristol BS8 1RJ, UK, and UMR CNRS 5276 Laboratoire de Géologie de Lyon, ENS Lyon, Université Lyon 1, 15, Parvis René Descartes, Lyon, France; Peggy Vincent [pvincent@mnhn.fr ], Staatliches Museum für Naturkunde, Stuttgart, Germany and CR2P, CNRS-MNHN-UPMC, Département Histoire de la Terre, Muséum National d'Histoire Naturelle, CP 38, 57 rue Cuvier, 75005 Paris, France; Guillaume Suan [guillaume.suan@univ-lyon1.fr] UMR CNRS 5276 Laboratoire de Géologie de Lyon, Université Claude Bernard Lyon 1 - Ecole Normale Supérieure Lyon, 2, rue Raphaël Dubois, Villeurbanne, France; Tom Sharpe [tom.sharpe1@me.com ], Peter Hodges [dr.p.hodges@geodrome.demon.co.uk], and Cindy Howells [Cindy.Howells@museumwales.ac.uk], Department of Geology, National Museum of Wales, Cathays Park, Cardiff CF10 3NP, UK; Matt Williams [Matt.Williams@brlsi.org], Bath Royal Literary and Scientific Institution, 16 Sueen Square, Bath BA1 2HN, UK; Valentin Fischer [v.fischer@ulg.ac.be], Geology Department, Université de Liège, B18, Allée du 6 Août, 4000 Liège, Belgium. 
This is an open-access article distributed under the terms of the Creative Commons

Attribution License (for details please see creativecommons.org), which permits unrestricted use, distribution, and reproduction in any medium, provided the original author and source are credited.

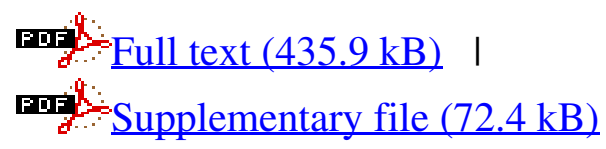

